\title{
Effect of the WWOX gene on the regulation of the cell cycle and apoptosis in human ovarian cancer stem cells
}

\author{
HONGCHAO YAN, JIANYE TONG, XIAOMAN LIN, QIUYU HAN and HONGXIANG HUANG \\ Department of Obstetrics and Gynecology, The Affiliated Hospital of Xuzhou Medical College, \\ Xuzhou, Jiangsu 221002, P.R. China
}

Received May 25, 2014; Accepted March 26, 2015

DOI: $10.3892 / \mathrm{mmr} .2015 .3640$

\begin{abstract}
In order to examine new ideas for gene therapy in ovarian cancer, the specific mechanism underlying the effects of the WW domain containing oxidoreductase (WWOX) gene on cell cycle regulation and apoptosis in human ovarian cancer stem cells was investigated. Ovarian cancer stem cells were transfected with a eukaryotic expression vector carrying the WWOX gene in vitro (recombinant plasmid) and cells transfected with the empty plasmid (empty plasmid) or untransfected cells were used as controls. Stably transfected cells were screened and amplified in culture and the WWOX protein was detected by western blot analysis in the three groups of cells. Western blot analysis was performed to detect the expression of cell cycle regulatory proteins cyclin E, cyclin-dependent kinase (CDK) 2, cyclin D1, CDK4 and apoptosis-related protein Wnt-5 $\alpha$ and c-Jun N-terminal kinase (JNK), while polymerase chain reaction (PCR) was used to detect alterations in the mRNA expression levels of caspase-3. The results demonstrated that the WWOX protein was stably expressed in cells of the recombinant plasmid group, but was not detected in cells of the empty plasmid group and the control group. Cell proliferation at each time point decreased significantly in the recombinant plasmid group compared with the empty plasmid group and the control group. Flow cytometric analysis demonstrated that the proportion of cells in the G0/G1 phase in the recombinant plasmid group was significantly higher than that of cells in the empty plasmid group and the control group. The rate of apoptosis in the recombinant plasmid group was significantly higher than that of cells in the empty plasmid group and the control group. Western blot analysis demonstrated that the expression levels of cyclin E, CDK2, cyclin D1 and CDK4 in the recombinant plasmid group were significantly lower
\end{abstract}

Correspondence to: Dr Hongchao Yan, Department of Obstetrics and Gynecology, The Affiliated Hospital of Xuzhou Medical College, 99 Huai Hai West Road, Xuzhou, Jiangsu 221002, P.R. China

E-mail: hongchaoyan@hotmail.com

Key words: WWOX gene, transfection, ovarian cancer, stem cells than those in the empty plasmid group and the control group; however, the expression levels of Wnt-5 $\alpha$ and JNK were significantly higher than those in the empty plasmid group and the control group. PCR results demonstrated that the mRNA expression level of caspase- 3 in the recombinant plasmid group was significantly higher than that in the empty plasmid group and the control group. In conclusion, the present study demonstrated that the WWOX gene can be stably expressed in ovarian cancer stem cells and that it inhibits the proliferation of ovarian cancer stem cells. The WWOX gene can downregulate the expression levels of cell cycle proteins cyclin E-CDK2 and cyclin D1-CDK4, which affects the cell cycle of ovarian cancer stem cells. Furthermore, the WWOX gene can upregulate the mRNA expression levels of Wnt-5 $\alpha$, JNK and caspase-3, thus contributing to apoptosis of ovarian cancer stem cells. The present study demonstrated that the WWOX gene may be an important molecular target for the treatment of ovarian cancer in the future.

\section{Introduction}

Ovarian cancer has the highest mortality rate worldwide among various types of gynecological malignancy and has become a serious threat to female health due to difficulty in early diagnosis and poor treatment (1). There are multiple types of ovarian cancer, however, epithelial ovarian cancer is the most common type and is also the most harmful (2). Several studies on gynecological cancer have aimed to understand the biological behavior and mechanism of ovarian cancer in more depth so that better treatments can be developed (3-5). In previous years, with the progress in the study of cancer stem cytology, the biological behavior of ovarian cancer has been recognized from a new perspective.

In 2000, Weissman first proposed the cancer stem cell theory (6), in which cancer is recognized as a disease of stem cells and cancer stem cells are a class of cells with self-renewal capacity, capable of unlimited proliferation and differentiation, and recognized as the principal cause of tumorigenesis, abnormal proliferation, metastasis and recurrence. During the study of tumor cells in ascites from patients with ovarian cancer, Bapat et al (7) found a number of sphere-forming cells capable of suspended growth. These sphere-forming cells have a strong cloning capability in vitro; they can self-renew and are capable of self-renewal and differentiation into 
tumors of the same nature when seeded into nude mice, thus confirming the presence of ovarian cancer stem cells (8). In addition to in vivo and in vitro experiments, our group applied paclitaxel to cells suspended in culture in serum-free medium containing epidermal growth factor (EGF), basic fibroblast growth factor (bFGF), Noggin and leukemia inhibitory factor (LIF) to successfully screen ovarian cancer stem cells, with characteristic expression of $\mathrm{CD}_{133^{+}}$and $\mathrm{CD} 117^{+}$, and identified their specific markers and biological characteristics (9). Our previous study laid a solid foundation for the present study.

The WW domain containing oxidoreductase (WWOX) gene was initially isolated and identified as a tumor suppressor gene in 2000 by Bednarek et al (10), spanning the entire autosomal fragile site FRA16D and promoting tumor progression through functional loss or protein inactivation. Gourley et al (11) demonstrated that the mRNA expression level of WWOX is significantly decreased in ovarian cancer cells compared with normal ovarian tissue, indicating that the WWOX gene can inhibit the occurrence of ovarian cancer.

To further investigate the effect of the WWOX gene on the biological behavior of ovarian cancer stem cells, the present study transfected ovarian cancer stem cells with the WWOX gene. The present study aimed to determine the effect of WWOX on the biological behavior of ovarian cancer stem cells and to identify the underlying mechanism in order to provide a theoretical basis for ovarian cancer gene therapy.

\section{Materials and methods}

Materials. Ovarian cancer stem cells and the pcDNA3.1-WWOX eukaryotic expression vector were provided by and stored at the Affiliated Hospital of Xuzhou Medical College (Xuzhou, China). The empty pcDNA3.1 plasmid was provided by Professor Shuqun $\mathrm{Hu}$ at the Research Center for Molecular Biology, Xuzhou Medical College. A liposome Lipofectamine 2000 transfection kit and G418 were purchased from Invitrogen Life Technologies (Carlsbad, CA, USA). Anti-WWOX (rabbit-anti-human monoclonal; 1:1,000; cat. no. 15800667461), cyclin E (goat-anti-rabbit monoclonal; 1:10,000; cat. no. 13764022678), cyclin-dependent kinase (CDK)2 (goat-anti-rabbit monoclonal; 1:10,000; cat. no. MAB4310), Wnt-5 $\alpha$ (goat-anti-rabbit monoclonal; 1:10,000; cat. no. MA1-12192), p-JNK (goat-anti-rabbit monoclonal; 1:10,000; cat. no. 254515), cyclin D1 (goat-anti-rabbit monoclonal; 1:10,000; cat. no. AM1125a) and CDK4 (goat-anti-rabbit monoclonal; 1:10,000; cat. no. AP1486c) primary and secondary antibodies were purchased from Chemicon (Billerica, MA, USA). Engreen Cell propidium iodide (PI), an Engreen cell cycle and an Engreen apoptosis detection kits were purchased from Nanjing KeyGen Biotech., Co., Ltd. (Nanjing, China). The present study was approved by the Ethics Committee of The Affiliated Hospital of Xuzhou Medical College.

Cell culture. Human ovarian cancer stem cells were cultured in serum-free medium containing EGF, bFGF, Noggin and LIF at $37^{\circ} \mathrm{C}, 5 \% \mathrm{CO}_{2}$ and saturated humidity in a closed thermostat incubator.

Transfection. The eukaryotic expression vector carrying the WWOX gene was transfected into ovarian cancer stem cells in vitro (recombinant plasmid), according to the manufacturer's instructions of the Lipofectamine 2000 transfection kit. Stably-transfected cells were screened and later expanded. Ovarian cancer stem cells transfected with the empty plasmid (empty plasmid) and untransfected cells (control group) were used as controls. Following transfection, cells were screened and passaged in selective medium containing neomycin.

Western blot analysis. The three groups of cells while in exponential growth phase were harvested and lysed in $200 \mu \mathrm{l}$ lysis buffer in ice. Following determining the protein concentration using a bicinchoninic acid assay kit (Sigma-Aldrich, St Louis, MO, USA), proteins were electrophoresed in $10 \%$ sodium dodecyl sulfate sodium-polyacrylamide gel and electrophoretically transferred onto nitrocellulose membranes. To detect WWOX protein, the membranes were blocked with $5 \%$ skim milk for 60 min, incubated with 1:1,000 anti-WWOX antibody (rabbit anti-human) at $4^{\circ} \mathrm{C}$ overnight and reacted with horseradish peroxidase-labeled goat anti-rabbit secondary antibody $(1: 10,000)$ at room temperature for $2 \mathrm{~h}$. Enhanced chemical fluorescent emission (LumiPico ${ }^{\circledR} \mathrm{ECL}$ reagent; Invitrogen Life Technologies) reagents were added and the membranes were exposed and developed with a highly sensitive X-ray film in a dark room. Other cell cycle and apoptotic proteins were detected similarly as described above.

MTT assay. As described above, the three groups of cells were seeded in 96-well plates at a density of $1.5 \times 10^{4}$ cells/ well. After 1, 2, 3, 4 and 6 days of culturing, $20 \mu \mathrm{l}$ MTT working solution was added and the cells were cultured for an additional $4 \mathrm{~h}$ in a $\mathrm{CO}_{2}$ incubator at $37^{\circ} \mathrm{C}$. Dimethyl sulfoxide was added to stop the reaction and each well was measured for the optical absorbance at $490 \mathrm{~nm}$ (A) on an enzyme-linked immunosorbent detector (SCJ204; Alpha Innotech Corp., San Diego, CA, USA). The data were used to draw a cell growth curve.

Determination of the cell cycle and apoptosis by flow cytometry. The three groups of cells were harvested and digested in $0.25 \%$ trypsin during the exponential growth phase. For cell cycle analysis, cells were washed with phosphate-buffered saline, centrifuged at 5,000 x g for $2 \mathrm{~min}$, fixed in $70 \%$ ethanol overnight, dispersed in $100 \mu \mathrm{l}$ phosphate salt buffer to produce a single cell suspension and analyzed by flow cytometry. For apoptosis analysis, cells were washed with phosphate-buffered saline, centrifuged at $5,000 \mathrm{x}$ g for $2 \mathrm{~min}$, adjusted to $1.5 \times 10^{5} \mathrm{cells} / \mathrm{ml}$, stained with $5 \mu \mathrm{l}$ annexin V-fluorescein isothiocyanate and $5 \mu \mathrm{l}$ PI for $10 \mathrm{~min}$ in the dark at room temperature and analyzed by flow cytometry.

Detection of caspase-3 $m R N A$ by reverse transcription quantitative polymerase chain reaction $(P C R)$. Total RNA was extracted from the three groups of cells during the exponential growth phase using TRIzol reagent. The reverse transcription reaction was performed according to the manufacturer's instructions (RT-PCR kit; Chemicon, Temecula, CA, USA). The following primer sequences were used: Caspase-3, forward 5'-GGTGTTGATGATGACATGGCG-3' and reverse 5'-GTACCCTCTGCAGCATGAGAGTAG-3' (amplified 
product: $419 \mathrm{bp}$ ); $\beta$-actin, forward 5'-TGGAGAAATCTGG CACCAC-3' and reverse 5'-GAGGCGTACAGGGATAGCAC-3' (amplified product: 295 bp). $\beta$-actin was used as an internal control. The reaction conditions were $94^{\circ} \mathrm{C}$ for $45 \mathrm{sec}, 55^{\circ} \mathrm{C}$ for $60 \mathrm{sec}$ and $72^{\circ} \mathrm{C}$ for $60 \mathrm{sec}$ for 30 cycles followed by extension at $72^{\circ} \mathrm{C}$ for $10 \mathrm{~min}$. The PCR-amplified products were electrophoresed on a $2 \%$ agarose gel, observed in a UV detector (4100; Olympus, Tokyo, Japan), photographed and recorded. The content of each amplified product was analyzed by the imaging software of an automatic gel electrophoresis analyzer Chemi Imager 5500 (Alpha Innotech Corp., San Diego, CA, USA). The content ratio of caspase- $3 \mathrm{mRNA} / \beta$-actin mRNA represents the relative mRNA expression level of caspase-3 .

Statistical analysis. The data are presented as the mean \pm standard error of the mean and were analyzed using SPSS 13.0 statistical software (SPSS, Inc., Chicago, IL, USA). The differences between two sets of data were compared by Student's t-test. Differences among multiple data were compared by one-way analysis of variance. $\mathrm{P}<0.05$ was considered to indicate a statistically significant difference.

\section{Results}

Alterations in the protein expression of WWOX in WWOX-transfected ovarian cancer stem cells. Western blot analysis results demonstrated that the WWOX protein was highly expressed in the recombinant plasmid group, but was not detected in the empty plasmid group or the control group (Fig. 1).

Alterations in cell proliferation in WWOX-transfected ovarian cancer stem cells. The results of the MTT assay demonstrated that after 1,2,3, 4, 5 and 6 days of culturing, the number of cells in the recombinant plasmid group was $0.425 \pm 0.022$, $0.471 \pm 0.032,0.525 \pm 0.023,0.741 \pm 0.023,0.899 \pm 0.018$ and $0.906 \pm 0.041$, respectively, which was significantly reduced compared with those in the control group and the empty plasmid group at each time point $(\mathrm{P}<0.05)$. No statistically significant difference was identified between the control group and the empty plasmid group ( $\mathrm{P}>0.05$; Fig. 2).

Alterations in the cell cycle in WWOX-transfected ovarian cancer stem cells. Flow cytometric analysis demonstrated that cells in the recombinant plasmid group were arrested at the G0/G1 phase and had a significantly reduced number of cells in the $\mathrm{S}$ phase compared with cells in the empty plasmid group and the control group. This difference was found to be statistically significant $(\mathrm{P}<0.05)$. However, no statistically significant difference was identified between the empty plasmid group and the control group ( $\mathrm{P}>0.05$; Table I).

Alterations in apoptosis in WWOX-transfected ovarian cancer stem cells. As shown by flow cytometry, the apoptotic rate in the recombinant plasmid group was $28.19 \%$, which was significantly higher compared with that in the empty plasmid group (8.27\%) and the control group $(7.11 \%$; $\mathrm{P}<0.05)$. No statistically significant difference was identified between the empty plasmid group and the control group (P>0.05; Fig. 3).
1 2 3

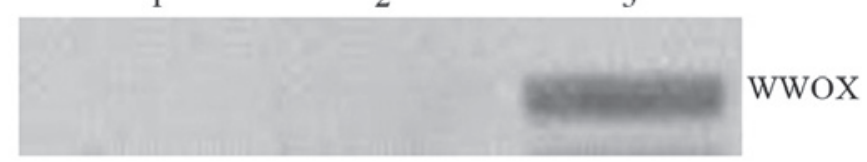

Figure 1. Detection of the WWOX protein in three groups of ovarian cancer stem cells by western blotting. 1, control group; 2 , empty plasmid group; 3 , recombinant plasmid group. WWOX, WW domain containing oxidoreductase.

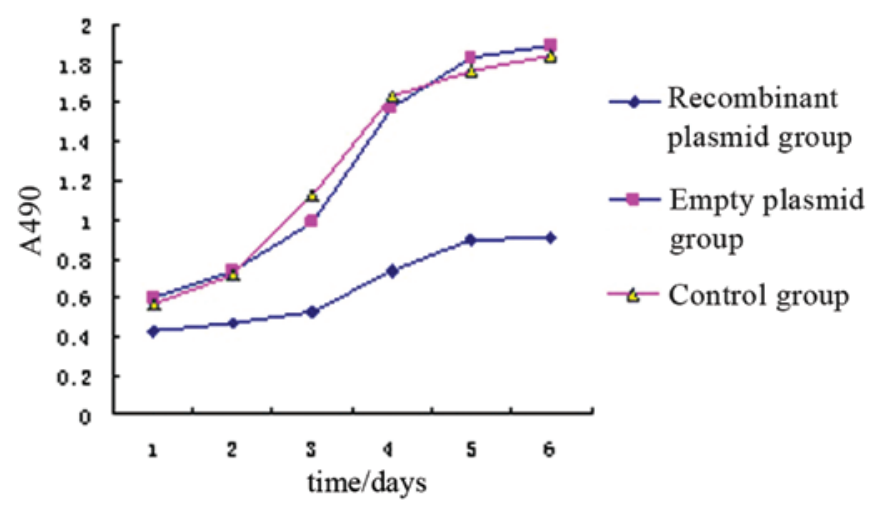

Figure 2. Growth curve of ovarian cancer stem cells following transfection of pcDNA3.1-WWOX.

Alterations in the expression of cell cycle regulatory proteins cyclin E, CDK2, cyclin D and CDK4 in WWOX-transfected ovarian cancer stem cells. The expression levels of cyclin E, CDK2, cyclin D1 and CDK4 in the recombinant plasmid group were: $1.033 \pm 0.012,0.945 \pm 0.037,1.068 \pm 0.057$ and $1.296 \pm 0.016$, respectively. These expression levels were significantly lower than that of the empty plasmid group $(1.551 \pm 0.035,2.547 \pm 0.026,1.607 \pm 0.045$, and $2.744 \pm 0.031$, respectively) and the control group $(1.546 \pm 0.015$, $2.568 \pm 0.044,1.601 \pm 0.028$, and $2.795 \pm 0.012$, respectively). These differences were statistically significant $(\mathrm{P}<0.05)$. However, no statistically significant difference was observed between the empty plasmid group and the control group (P>0.05; Fig. 4).

Alterations in apoptosis-associated factors Wnt-5 $\alpha$ and JNK and the mRNA expression of caspase-3 in WWOX-transfected ovarian cancer stem cells. Western blotting results demonstrated that the recombinant plasmid group had significantly higher protein levels of Wnt-5 $\alpha$ and JNK $(1.539 \pm 0.055$ and $1.565 \pm 0.042$, respectively) compared with the empty plasmid group $(0.816 \pm 0.021$ and $1.087 \pm 0.035$, respectively) and the control group $(0.849 \pm 0.007$ and $1.085 \pm 0.026$, respectively). The differences were statistically significant $(\mathrm{P}<0.05)$. No statistically significant difference was identified between the empty plasmid group and the control group ( $\mathrm{P}>0.05$; Fig. 5).

The mRNA expression level of caspase-3 in the recombinant plasmid group was significantly higher than that in the empty plasmid group and the control group $(\mathrm{P}<0.05)$. While no statistically significant difference was identified between the empty plasmid group and the control group ( $P>0.05$; Fig. 6). 
Table I. Cell cycle in each group of cells following transfection of the WWOX gene $(\bar{x} \pm$ standard deviation, $\%)$.

\begin{tabular}{lccc}
\hline Group & $\mathrm{G}_{0} / \mathrm{G}_{1}$ phase & $\mathrm{S}$ phase & $\mathrm{G}_{2} / \mathrm{M}$ phase \\
\hline Recombinant plasmid & $65.07 \pm 0.60$ & $27.52 \pm 0.60$ & $7.61 \pm 0.18$ \\
Empty plasmid & $33.87 \pm 0.84$ & $58.03 \pm 0.72$ & $8.15 \pm 0.54$ \\
Blank control & $33.69 \pm 1.28$ & $57.87 \pm 0.71$ & $8.24 \pm 0.31$ \\
\hline
\end{tabular}

WWOX, WW domain containing oxidoreductase.
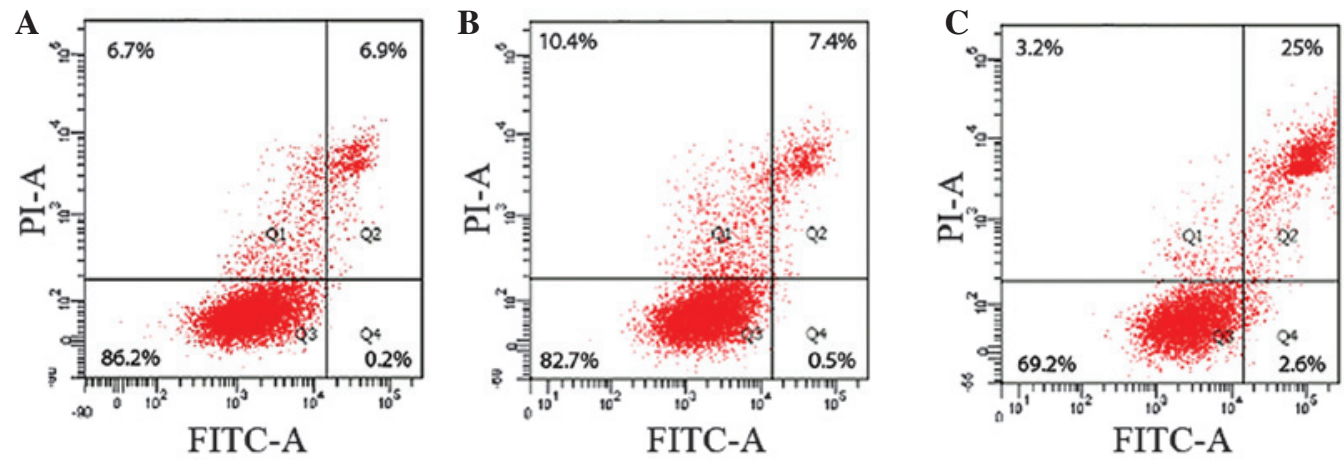

Figure 3. Apoptosis of three groups of cells following transfection of the WWOX gene. (A) Control group; (B) empty plasmid group; (C) recombinant plasmid. FITC, fluorescein isothiocyanate.

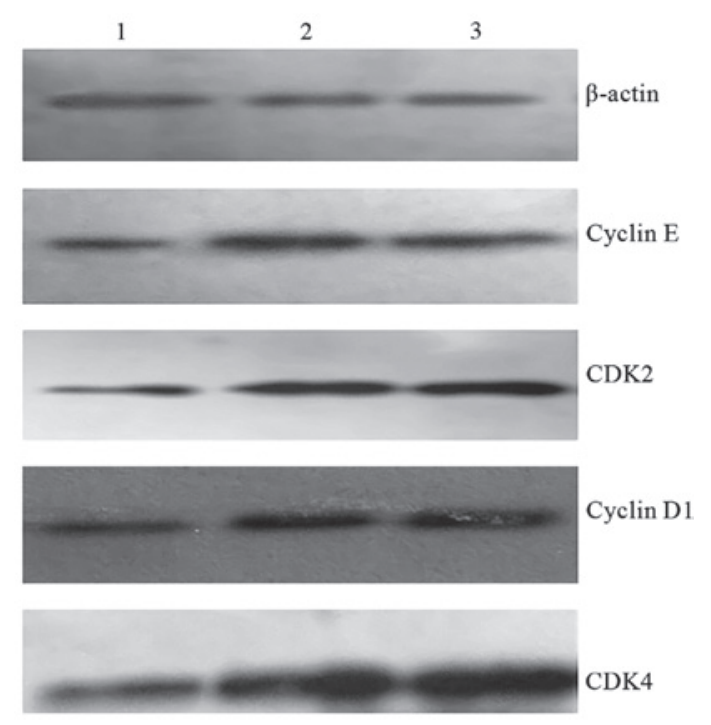

Figure 4. Detection of protein cyclin E, CDK2, cyclin D1 and CDK4 in three groups of cells by western blotting. 1, recombinant plasmid group; 2, empty plasmid group; 3 , control group. CDK2, cyclin-dependent kinase 2 .

\section{Discussion}

Ovarian cancer is one of the most common types of female genital tumors worldwide. Due to the difficulty of early diagnosis in ovarian cancer, $60-70 \%$ of patients are diagnosed at an advanced phase (12). In addition, with poor treatment efficacy for advanced cases, ovarian cancer ranks first in gynecological cancer-associated mortality and is a severe threat to female health (13).

Cancer stem cells are a class of multipotent cells capable of self-renewal, unlimited proliferation and differentiation;

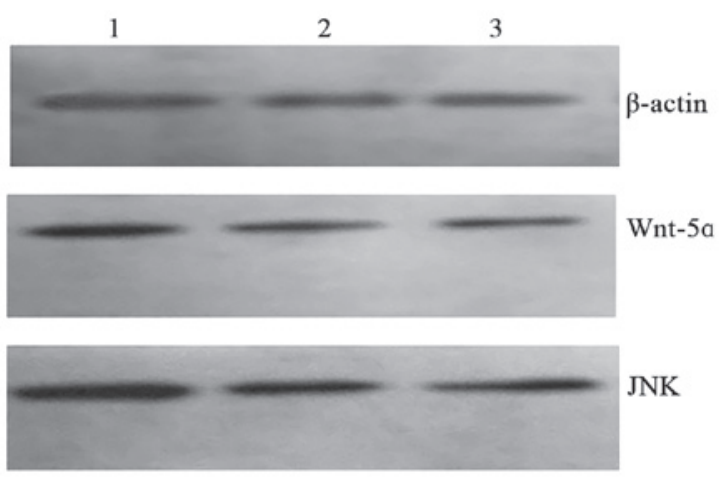

Figure 5. Detection of Wnt-5 $\alpha$ and p-JNK protein in three groups of cells by western blotting. 1, recombinant plasmid group; 2, empty plasmid group; 3 , control group. JNK, c-Jun N-terminal kinase.

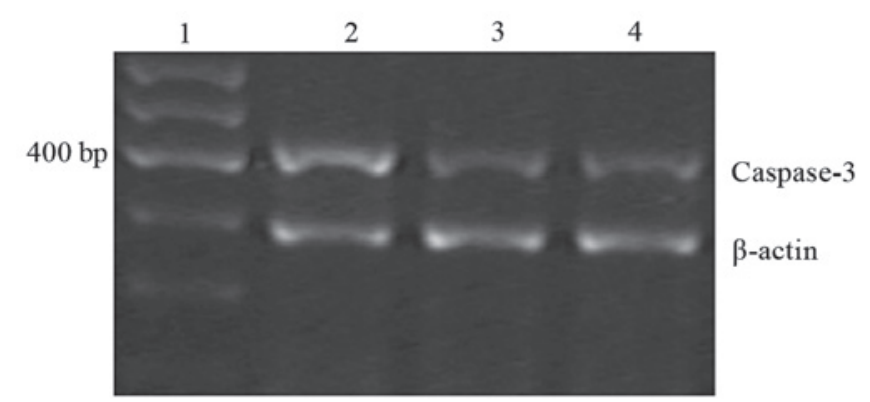

Figure 6. Detection of caspase-3 mRNA in three groups of cells by polymerase chain reaction. 1 , marker; 2 , recombinant plasmid group; 3 , empty plasmid group; 4 , control group.

however, they account for $<1 \%$ of total tumor cells (14). During the cancer stem cell proliferation process and through uneven 
division, cancer stem cells divide to form a new tumor stem cell and another cell that may eventually differentiate into daughter cells of various types of cells, including tumor cells. The result of this process is the maintenance of a stable number of cancer stem cells and also the formation of a tumor (15). As increasing evidence supports the cancer stem cell theory (16-18), it has been hypothesized that ovarian cancer may also be a disease of stem cells. Zhang et al (19) successfully isolated ovarian cancer initiating cells from human serous ovarian cancer specimens and found that these cells can self-renew, form a suspension sphere and differentiate into tumors of the same nature when these sphere-forming cells were inoculated into mice in vivo, thus confirming the presence of ovarian cancer stem cells in ovarian cancer.

The WWOX protein contains two WW domains at the $\mathrm{N}$-terminus (double tryptophan domain) that can easily bind proline-rich structures to participate in protein-protein interactions and one short-chain dehydrogenase/reductase domain at the C-terminus (oxidoreductase area) (20). Several studies have demonstrated that the WWOX gene is important in malignant tumors, particularly in the development of hormone-dependent tumors (21-23). One study demonstrated that normal ovarian tissue expressed high levels of the WWOX protein, however, the expression was reduced or absent in ovarian cancer patients; furthermore, a loss of WWOX expression was detected in $70 \%$ of ovarian mucinous adenocarcinoma and $42 \%$ of clear cell carcinoma (24). A previous study also found that epithelial ovarian carcinoma exhibited low expression levels of WWOX mRNA and in epithelial ovarian cancer and ovarian borderline tumor tissues, the positive expression rate and mRNA expression levels of WWOX were significantly lower than that in benign ovarian tumors and normal ovarian tissues (25). To further investigate the effect of the WWOX gene on the biological behavior of ovarian stem cells, the present study transfected ovarian cancer stem cells with WWOX by using the eukaryotic expression carrier pcDNA3.1-WWOX and detected cell proliferation by using an MTT assay in WWOX-transfected ovarian cancer stem cells. The results demonstrated that the WWOX gene can be stably expressed in ovarian cancer stem cells and inhibits the proliferation of ovarian cancer stem cells.

Subsequently, flow cytometry was used to investigate the cell cycle of ovarian cancer stem cells. The results demonstrated that following transfection of the WWOX gene, the cell cycle of ovarian cancer stem cells was arrested in the G0/G1 phase, indicating that the WWOX gene significantly inhibits the cell cycle of ovarian cancer stem cells.

The study by Xue et al (26) suggests that cancer is a disease of the cell cycle and the occurrence of tumors is closely associated with abnormal cell cycle regulation, where cyclin E-CDK2, cyclin D1-CDK4 and other cell cycle regulatory proteins are involved. Cyclin $\mathrm{E}$ is located on human chromosome $19 \mathrm{q} 12-13$ and encodes a $50 \mathrm{kDa}$ protein containing a cyclin box to interact with CDKs. Cyclin-dependent kinase 2 (CDK2) is located on human chromosome 12q13 and encodes a $33 \mathrm{kDa}$ protein of a threonine kinase. Cyclin E and CDK2 are important regulatory factors in the $\mathrm{Gl}$ and $\mathrm{S}$ phases of the cell cycle (27). Cyclin E binds to CDK2 to form the cyclin E-CDK2 complex, which is a key kinase complex and is important in transition from the $\mathrm{G} 1$ to $\mathrm{S}$ phase. Expression abnormalities in cyclin $\mathrm{E}$ and $\mathrm{CDK} 2$ are closely associated with the occurrence and development of breast cancer, stomach cancer, colon cancer and other types of tumor $(28,29)$.

The cyclin D1 gene is located on the long arm of human chromosome 11, area 13 (11q13). In mid-G1 phase, the expressed cyclin Dl protein binds CDK4 to activate and maintain CDK4 activity and promotes cell cycle progression (30). The CDK4 gene is located on the long arm of human chromosome 12, areas 13-14 (12q13-14) and is the regulatory center for the entry of G1 to S phase. When cyclin D1 binds $\mathrm{CDK} 4, \mathrm{Rb}$ kinase is activated to phosphorylate $\mathrm{Rb}$ protein, and hyperphosphorylation of $\mathrm{Rb}$ protein exerts its transcriptional effect following conformational changes, promoting cells to complete transition of $\mathrm{Gl} / \mathrm{S}$ phase and to enter the proliferative state (31).

To further investigate the effect and mechanism of the WWOX gene on the cell cycle of ovarian cancer stem cells, the present study used western blot analysis to detect cell cycle-associated proteins in ovarian cancer stem cells. The results demonstrated that following transfection of the WWOX gene, ovarian cancer stem cells expressed significantly lower levels of cyclin E, CDK2, cyclin D1 and CDK4 proteins than the empty plasmid group and the control group. These results indicate that the WWOX gene may downregulate the expression levels of cyclin E-CDK2 and cyclin D1-CDK4 affecting the cell cycle of ovarian cancer stem cells.

Subsequently, flow cytometry was used to detect apoptosis in ovarian cancer stem cells. The results demonstrated that following transfection of the WWOX gene, the rate of apoptosis in ovarian cancer stem cells was significantly increased. These data indicate that the WWOX gene can promote apoptosis of ovarian cancer stem cells.

A previous study demonstrated that Wnt- $5 \alpha$, JNK and caspase-3 are important in the apoptotic process of tumor cells (32). The Wnt signaling pathway induces apoptosis in a variety of tumor stem cells in vivo, regulates the proliferation of a variety of tumor stem cells and is important in tumorigenesis and development. Currently, the canonical Wnt signal transduction pathways is being extensively investigated and is considered to be a major factor affecting tumor stem cells. However, studies by McDonald et al (33) and Nomachi et al (34) suggest that the JNK signal transduction pathway is also closely associated with the occurrence and development of tumor stem cells and the non-canonical Wnt signaling pathway mediated by the JNK pathway also has significant effects on tumor stem cells. The Wnt/JNK signaling pathway is important in multiple processes, including cell proliferation, promotion of apoptosis, mediation of the inflammatory response and gene expression. Caspase- 3 is a convergent point of signal delivery from multiple apoptotic stimuli and is considered to be the most important and effective caspase. Caspase 3 is a downstream substrate of the apoptotic Wnt/JNK signaling pathway and its activation is a sign of irreversible apoptosis $(35,36)$.

Therefore, in order to further examine the mechanism underlying the effect of WWOX on the apoptosis of ovarian stem cells, western blot analysis was used to assess apoptosis-associated factors in ovarian cancer stem cells. The results demonstrated that following transfection of the WWOX gene, ovarian cancer stem cells expressed significantly higher levels 
of Wnt- $5 \alpha$, JNK and caspase-3 mRNA, indicating activation of the Wnt/JNK/caspase-3 signaling pathway. Furthermore, the WWOX gene may promote apoptosis of ovarian cancer stem cells by upregulating the expression levels of Wnt-5 $\alpha, \mathrm{JNK}$ and caspase-3.

In the present study, the experimental results suggest that the WWOX gene may downregulate the protein expression levels of cyclin E-CDK2 and cyclin D1-CDK4 to affect cell proliferation and the cell cycle of ovarian cancer stem cells. By contrast, the WWOX gene may upregulate the expression levels of Wnt- $5 \alpha$, JNK and caspase-3 to promote apoptosis of ovarian cancer stem cells. Further studies are required to elucidate what the specific target of the WWOX gene is and its role in regulating cell cycle and apoptosis of ovarian cancer stem cells. These findings demonstrated that the WWOX gene may be an important molecular target for the treatment of ovarian cancer in the future

\section{References}

1. Lim W and Song G: Discovery of prognostic factors for diagnosis and treatment of epithelial-derived ovarian cancer from laying hens. J Cancer Prev 18: 209-220,2013.

2. Desai A, Xu J, Aysola K, et al: Epithelial ovarian cancer: An overview. World J Transl Med 3: 1-8, 2014.

3. Zeimet AG, Reimer D, Sopper S, et al: Ovarian cancer stem cells. Neoplasma 59: 747-755, 2012.

4. Yang L and Lai D: Ovarian cancer stem cells enrichment. Methods Mol Biol 1049: 337-345, 2013.

5. Dunlop CE, Telfer EE and Anderson RA: Ovarian stem cells - potential roles in infertility treatment and fertility preservation. Maturitas 76: 279-283, 2013

6. Weissman IL: Stem cells: units of development, units of regeneration and units in evolution. Cell 100: 157-168, 2000.

7. Bapat SA, Mali AM, Koppikar CB, et al: Stem and progenitor-like cells contribute to the aggressive behavior of human epithelial ovarian cancer. Cancer Res 65: 3025-3029, 2005.

8. Ponnusamy MP and Batra SK; Ovarian cancer: Emerging concept on cancer stem cells. J Ovarian Res 1: 4, 2008.

9. Yan HC, Yu N, Tong JY, et al: Isolation of cancer stem cells from ovarian cancer cell line HO9810 and identification of their biological characteristics. Jiangsu Medical Journal 10: 1152-1155, 2012 (In Chinese).

10. Bednarek AK, Laflin KJ, Daniel RL, et al: WWOX, a nove WW domain-containing protein mapping to human chromosome16q23.3-24.1, a region frequently affected in breast cancer. Cancer Res 60: 2140-2145, 2000.

11. Gourley C, Paige AJ, Taylor KJ, et al: WWOX mRNA expression profile in epithelial ovarian cancer supports the role of WWOX variant 1 as a tumour suppressor although the role of variant 4 remains unclear. Int J Oncol 26: 1681-1689, 2005.

12. Burges A and Schmalfeldt B; Ovarian cancer: Diagnosis and treatment. Dtsch Arztebl Int 108: 635-641, 2011.

13. Jemal A, Siegel R, Ward E, et al: Cancer statistics 2009. CA Cancer J Clin 59: 225-249, 2009.

14. Kopper L and Hajdú M: Tumor stem cells. Pathol Oncol Res 10: 69-73, 2004.
15. Gao Q, Geng L, Kvalheim G, et al: Identification of cancer stem-like side population cells in ovarian cancer cell line OVCAR-3. Ultrastruct Pathol 33: 175-181, 2009.

16. Dyall S, Gayther SA and Dafou D. Cancer stem cells and epithelial ovarian cancer. J Oncol 2010: 105269, 2010.

17. Conic I, Dimov I, Tasic-Dimov D, et al: Ovarian epithelial cancer stem cells. Scientific World Journal 11: 1243-1269, 2011.

18. Gangemi R, Paleari L, Orengo AM, et al: Cancer stem cells: a new paradigm for understanding tumor growth and progression and drug resistance. Curr Med Chem 16: 1688-1703, 2009.

19. Zhang S, Balch C, Chan MW, et al: Identification and characterization of ovarian cancer-initiating cells from primary human tumors. Cancer Res 68: 4311-4320, 2008.

20. Aqeilan RI, Pekarsky Y, Herrero JJ, et al: Functional association between Wwox tumor suppressor protein and p73, a p53 homolog. Proc Natl Acad Sci USA 101: 4401-4406, 2004.

21. Chang NS, Schultz L, Hsu LJ, et al: $17 \beta$-Estradiol upregulates and activates WOX1/WWOXv1 and WOX2/WWOXv2 in vitro: potential role in cancerous progression of breast and prostate to a premetastatic state in vivo. Oncogene 24: 714-723, 2005.

22. Aqeilan RI, Trapasso F, Hussain S, et al: Targeted deletion of Wwox reveals a tumor suppressor function. Proc Natl Acad Sci USA 104: 3949-3954, 2007.

23. Paige AJ, Taylor KJ, Taylor C, et al: WWOX: a candidate tumor suppressor gene involved in multiple tumor types. Proc Natl Acad Sci USA 98: 11417-11422, 2001.

24. Nunez MI, Rosen DG, Ludes-Meyers JH, et al: WWOX protein expression varies among ovarian carcinoma histotypes and correlates with less favorable outcome. BMC Cancer 5: 64, 2005.

25. Yan HC, Lu XY and Han QY: WWOX mRNA expression in epithelial ovarian cancer and its clinical significance. Acta Academiae Medicinae Xuzhou 2: 126-128, 2007 (In Chinese).

26. Xue Q, Sano T, Kashiwabara K, et al: Aberrant expression of $\mathrm{pRb}$, p16, p14ARF, MDM2, p2l and p53 in stage I adenocarcinomas of the lung. Pathal Int 52: 103-109, 2002.

27. Graña X and Reddy EP: Cell cycle control in mammalian cells: Role of cyclins, cyclin dependent kinases (CDKs), growth suppressor genes and cyclin-dependent kinase inhibitors (CDKIs). Oncogene 11: 211-219, 1995.

28. Xiangming $C$, Natsugoe $S$, Takao $S$, et al: The cooperative role of p27 with cyclin $\mathrm{E}$ in the prognosis of advanced gastric carcinoma. Cancer 89: 1214-1219, 2000.

29. Kim JH, Kang MJ, Park CU, et al: Amplified CDK2 and cdc2 activities in primary colorectal carcinoma. Cancer 85: 546-553, 1999.

30. Hartwell LH and Kastan MB: Cell cycle control and cancer. Science 266: 1821-1828, 1994.

31. Ayad NG: CDKs give Cdc6 a license to drive into $\mathrm{S}$ phase. Cell 122: 825-827, 2005.

32. Oishi I, Suzuki H, Onishi N, et al: The receptor tyrosine kinase Ror2 is involved in non-canonical Wnt5a/JNK signalling pathway. Genes Cells 8: 645-654, 2003.

33. McDonald SL and Silver A: The opposing roles of Wnt-5a in cancer. Br J Cancer 101: 209-214, 2009.

34. Nomachi A, Nishita M, Inaba D, et al: Receptor tyrosine kinase Ror2 mediates Wnt5a-induced polarized cell migration by activating c-Jun $\mathrm{N}$-terminal kinase via actin-binding protein filamin A. J Biol Chem 283: 27973-27981, 2008.

35. Rana SV: Metals and apoptosis: recent developments. J Trace Elem Med Biol 22: 262-284, 2008.

36. Abi-Hachem RN, Zine A and Van De Water TR: The injured cochlea as a target for inflammatory processes, initiation of cell death pathways and application of related otoprotectives strategies. Recent Pat CNS Drug Discov 5: 147-163, 2010. 